Recibido: 16-04-2014

Aceptado: 20-08-2014

\title{
Lateralidad zurda, un problema y una solución.
}

\section{Left-handed laterality, a problem and a solution}

\section{Carmen Leni Alvarez Taco}

RESUMEN. En el presente artículo se brinda una revisión teórica de la situación histórico-social y educativa de las personas con lateralidad zurda, así como el proceso de formación de la lateralidad, lo cual sirvió de base para la realización de un estudio correlacional entre la comprensión lectora y el autoconcepto de escolares zurdos de segundo y tercer grado de primaria, asimismo, se compararon dichas variables ente escolares zurdos y diestros. Estos resultados también se presentan en este artículo.

La condición de zurdo ha sido vista, a lo largo de la historia humana, como algo negativo e incluso peligroso, de tal forma que se realizaban toda clase de esfuerzos por cambiar esta situación en el niño, contraviniendo con ello su verdadera naturaleza, ya que la lateralidad zurda tiene una base genética que explica su expresión. Sin embargo, ya que los zurdos equivalen aproximadamente al $10 \%$ de la población mundial, es un grupo humano al cual se le ha prestado poca atención, y por ende, los problemas que surgen en ellos son los que menos atención obtienen, generando así la necesidad de desarrollar sus propias estrategias adaptativas que le permitan una adecuada inserción a un entorno social contrario a su naturaleza.

Palabras clave: lateralidad, lateralidad zurda, comprensión lectora. Keywords: laterality, lefthanded laterality, reading comprehensión.

Carmen Leni Alvarez Taco

UNMSM leni_alvareztaco@hotmail.
ABSTRACT. In this article, a theoretical review of educational and historicalsocial status of people with left-handed laterality is provided as well as formation process of laterality, which served as the basis for carrying out of a correlational study between reading comprehension and self-concept of left-handed schoolchildren in the second and third grade of primary education, likewise variables between left-handed and right-handed schoolchildren are compared. These results in this article are also presented.

The condition of left-hander has been seen, throughout human history, as something negative and even dangerous, so that all the efforts were made to change this situation in the child, contravening with this to their true nature, since left-handed laterality has a genetic basis that explains her expression. However, since left-hander approximately are equivalent to $10 \%$ of the world population, it is a human group that has received little attention, and therefore, their problems that arise in them are the least underserved, generating thus the need to develop their own adaptive strategies that allow to them a proper insertion to a social environment contrary to his nature. 


\section{UNIFÉ - EPG}

\section{Introducción}

A

lo largo de la historia el concepto de zurdo ha sido y es asociado a aspectos negativos tales como: lisiado, desdichado, oprimido, dolencia, defecto, malintencionado, mal modo, torcido, no recto, inclinación a lo malo, viciado, infeliz y hasta funesto, es decir, ha representado todo lo nocivo y perjudicial en la vida del hombre, mientras que la derecha se ha relacionado con lo positivo y el bien. Esto ha generado el uso común de frases como: $\mathrm{Me}$ levanté con el pie izquierdo (justificación de lo mal que le va en el día), no vayas por el camino de la izquierda (recomendación para hacer cosas positivas), o el diablo se encuentra a tu izquierda (mensaje utilizado comúnmente por las madres para que sus hijos les hagan caso); todas ellas resaltan la connotación negativa del lado izquierdo. Pero esto no sólo ocurre en el Perú, también en Cuba, por ejemplo, es muy común que cuando se comete un error se le denomine al hecho una zurdada, es decir, ser zurdo es una equivocación (AUPEC, 1996). Por el contrario, en alusión al lado derecho, las frases más conocidas son las siguientes: Le estrecho la derecha (como señal de haber realizado un buen negocio), un hombre hecho y derecho (es una persona madura y correcta), es mi mano derecha (para referirse a la persona de confianza), entre otras frases conocidas.

Esta forma de conceptuar todo lo relacionado a la zurdera tuvo como consecuencia por muchas décadas que los zurdos sean considerados como seres inferiores y como tales fueran víctimas de la discriminación y el rechazo de los diestros; sin embargo, a lo largo de la historia de la humanidad, la capacidad de los zurdos se ha hecho evidente a través de las acciones de zurdos célebres que permiten -en la actualidad- promover un replanteamiento de su posición.

\section{Los Zurdos en la Prehistoria}

En principio, es necesario recordar que aún se desconocen las razones reales por las cuales se da la especialización lateral en el ser humano, e incluso las teorías científicas existentes no explican con certeza esta tendencia. Pestana-Tirado, Moreno y Di Filippo (2004) mencionan que la simetría motora de las extremidades anteriores, no se encuentra en los prosimios, primates menores ni póngidos en su estado natural y supone que tal vez se deba a que las demandas adaptativas en su ambiente natural no lo exigieron. En tiempos prehistóricos en el género Homínido, específicamente en el Homo habilis, hace más de dos millones de años en el paleolítico, primeras fases de la edad de piedra, cuando predominaba una cultura basada en la caza, pesca y recolección, las cuales, sin dejar de ser simples, eran realizadas por cada individuo según su conveniencia, sin reglas sociales y sin preferencia manual definida (Holder, 2004).

Los diferentes hallazgos arqueológicos de la edad de piedra han demostrado que las herramientas utilizadas por el hombre prehistórico han estado divididas igualmente para el uso de ambas manos, sin embargo, los estudios de los dibujos de las cuevas europeas han indicado una preferencia por la mano izquierda, lo que podría estar evidenciando que el uso de la mano izquierda era tan o más común que el uso de la mano derecha, por lo cual se puede suponer que no estaba aún establecido el dominio o preferencia lateral ya sea diestra o zurda. En América del Sur, uno de los yacimientos arqueológicos más importantes es la Cueva de las Manos, en Santa Cruz-Argentina, la cual cuenta en sus paredes con pinturas rupestres, algunas de casi diez mil años de antigüedad en las que se superponen manos negras, verdes, ocres, rojas, amarillas y violetas (entre otras figuras) contándose algo más de ochocientas impresiones de manos, casi todas zurdas y realizadas en negativo. Las pinturas fueron estampadas por lo menos en tres etapas por los antecesores de los tehuelches, que van desde los nueve mil quinientos años de antigüedad hasta los mil quinientos (Quintans, 2005).

La domesticación de animales, la agricultura, la manufactura de vasijas y textiles que seiniciaen elneolítico (etapas tardías de la edad de piedra) y la consiguiente necesidad de elaborar herramientas más sofisticadas, podría haber orientado al uso o preferencia -aleatoria o por accidente- de una de las manos. Posteriormente 
en la edad de bronce, al iniciar la fabricación de armas y otros utensilios metálicos, las tareas se tornaron más complejas, y entonces la preferencia manual perdió su carácter fortuito y se dio la prioridad de la mano derecha como rasgo biológico distintivo de nuestra especie (Pestana-Tirado et al., 2004).

\section{Los Zurdos en la Historia}

Aparentemente, la preferencia por el uso de la mano derecha fue condicionada por una serie de actividades cotidianas que realizaba el hombre al dejar su vida nómada, lo que ha generado una serie de suposiciones que podrían explicar las causas de esta especialización, por ejemplo, para los editores de Cebec (2004), la preferencia por el lado derecho pudo haberse originado por la adoración del sol, es decir, para que una persona -encontrándose en el hemisferio norte- pueda seguir el sol, tiene que hacerle frente al sur, y por tanto, moverse de izquierda a derecha hasta la puesta de sol en el oeste, dándole al lado derecho y a la mano derecha una gran importancia.

A finales del siglo XIX, el historiador inglés y zurdo Thomas Carlyle hizo un intento por explicar porqué el número de diestros es tan superior al de zurdos, y para él, en la antigüedad los hombres protegían su corazón colocando su escudo en el lado izquierdo y por lo tanto, cualquier arma tuvo que ser sostenida con la mano derecha, la que se convirtió con el tiempo en la mano dominante.

Otro aspecto importante en la determinación de la preferencia manual a lo largo de la historia, fue la posición de la Iglesia Católica frente a los zurdos a quienes declaró sirvientes del demonio, privilegiando el uso de la mano derecha incluso en los signos y señales propios de dicha religión, así se tiene que precisamente los simbolismos más utilizados por lo católicos se basan fuertemente en el uso de la mano derecha: sólo con esta mano se da la bendición y se hace la señal de la cruz. Esto generó que los zurdos fueran obligados a convertirse en diestros en las escuelas católicas, por las razones ya explicadas.

En un artículo publicado por AUPEC en 1996 se menciona que en la cultura árabe la mano derecha se utiliza para escribir, rendir culto a Dios y en general para las cosas positivas o buenas, mientras que la mano izquierda, en cambio, es utilizada para las cosas malas.

Como se puede observar, la percepción negativa de la zurdera no sólo es evidenciable en las definiciones o en las conceptualizaciones manejadas, sino también se vieron traducidas en actitudes, ejemplos de esto son los presentados en las páginas web de Cebec (2004) y de Segovia (2004), que a continuación se presenta:

En la Edad Media, los zurdos eran presa favorita para los inquisidores pues consideraban que tenían antecedentes de brujos o demoníacos y muchos fueron quemados; el anillo de casado se lleva en la mano izquierda para espantar a los malos espíritus que pueden deshacer el matrimonio; los nativos de Nueva Guinea nunca tocan con su dedo pulgar izquierdo los vasos, por la creencia de que podrían envenenar los brebajes que contiene; las mujeres Maoríes, ondean sus ropas matrimoniales con la mano derecha ya que la mano izquierda podría profanar sus ropas (la consecuencia de usar la mano izquierda es la muerte); las tribus africanas de las orillas del río Níger no dejan que sus mujeres preparen la comida con su mano izquierda por miedo a la magia negra; en el Japón, hasta hace pocas décadas, la zurdera de una esposa era suficiente motivo para solicitar el divorcio; en una época, cuando la policía buscaba criminales, siempre comenzaba sus investigaciones con los zurdos.

En cuanto a la Educación, hasta hace algunas décadas se tomaban medidas drásticas, como atar la mano izquierda del niño a la espalda para que escriba con la derecha, e incluso actualmente se evidencia que no existe una preparación especial de las y los docentes de inicial y primaria para atender a estos niños, a pesar que la/el docente requiere de guiar los primeros trazos y apoyar las actividades de coordinación visomotora (importantes para el logro de los aprendizajes, sobretodo de la lectoescritura), lo cual se hace muy difícil si se considera que nuestros maestros son, en su gran mayoría, diestros.

Como se puede observar, esto ha generado que por mucho tiempo, la población infantil zurda haya formado parte del grupo de niños con problemas de aprendizaje -relacionados con su lateralidad- por lo 


\section{UNIFÉ - EPG}

cual, se tomaban como medidas correctivas algunas de las siguientes opciones: a) Estimular la realización de actividades de coordinación motora fina con la mano derecha; b) Seguir utilizando la mano izquierda con apoyo de especialistas; c) Dejar que el niño desarrolle por él mismo estrategias de aprendizaje condicionadas a su lateralidad; o d) Retirarlo de la escuela por mal aprovechamiento.

\section{Proceso de formación de la lateralidad}

Pese a las dificultades para determinar el inicio de la lateralización, ésta es entendida como la consecuencia de un proceso de desarrollo del sistema nervioso, el cual permite elevar el grado de complejidad funcional del cerebro, produciéndose así la especialización de un lado del cuerpo, definido básicamente por una mayor destreza de la mano y pie, así como una mayor agudeza visual y auditiva de uno de los dos lados, permitiéndose así definir a los diestros como aquellas personas que muestran una mayor habilidad del hemicuerpo derecho, y a los zurdos como aquellas personas que muestran una mayor habilidad del hemicuerpo izquierdo. En este sentido, los investigadores coinciden al señalar que el predominio funcional de un lado del cuerpo se determina, no por la educación, sino por la supremacía de un hemisferio cerebral sobre el otro, por lo tanto, el ser diestro o zurdo dependería básicamente de dos factores: primero de la herencia y luego del adiestramiento o experiencia que cada niño tiene durante su maduración cerebral.

Especificando aún más, cuando se dice que un niño es zurdo, se está haciendo mención a la llamada lateralizacióncerebral o dominanciacerebral, lo que significa que de los dos hemisferios en que está dividido el cerebro -los cuales no son exactamente iguales como se verá posteriormente- tiene un predominio cerebral del hemisferio derecho, y si se toma en consideración que el sistema nervioso es cruzado, el predominio funcional de su cuerpo será el izquierdo, evidenciándose esto en el uso de la mano, ojo, oído y pie izquierdo, ya que son más hábiles o fuertes con ellos.

Además de la lateralización zurda o diestra, también se puede dar la denominada lateralización cruzada, la cual hace referencia a aquellos niños que, por ejemplo, son diestros de mano y zurdos de ojo o pie, y viceversa, y aunque se cree que sólo el 10\% de la población es zurda (aunque otros investigadores hacen mención a porcentajes mayores llegando hasta el 15\%), se dice que en realidad sólo el $65 \%$ de la población son diestros verdaderos, dado que son bastante frecuentes los casos de lateralidad cruzada, manifestada con predominio de mano derecha y pie izquierdo o viceversa (Zuliani, 2003).

La especialización de los hemisferios cerebrales es una función que va madurando y emergiendo a medida que ocurren los cambios estructurales del sistema nervioso propios del desarrollo infantil, y la especialización de un hemisferio cerebral en tareas de control motor fino de la mano y en habilidades de lenguaje, llevará a mayores logros funcionales (Cebec, 2004).

Según Förster, la evolución normal del cerebro busca que el ser humano tenga una lateralidad homogénea (ojo, oído, mano y pie dominantes del mismo lado del cuerpo), por lo que su formación pasa por una serie de etapas hasta completarse (Cebec, 2004), así se tiene que, un niño menor de un año aún no logra definir su lateralización, es decir, es ambidiestro (no tiene preferencia manual definida, desarrollando con ambas manos la misma habilidad y con la misma eficiencia), pero entre los dos y cuatro años de edad (aproximadamente a los tres años), ya logra establecer la preferencia definitiva de una mano sobre la otra, aunque suela probar ambas manos en numerosas ocasiones, es por esto que el aprestamiento -y la educación psicomotriz en general- que se da en la enseñanza del nivel inicial, suele ser un factor determinante para la definición de la lateralidad del niño, toda vez que en esta etapa se refuerza el dominio preferente de un lado del cuerpo, y sobre todo de la dominancia manual a través de sus primeros trazos.

Si se toma en consideración que el cerebro se mantiene como un órgano plástico, cuyo desarrollo, organización e integración neurológica continúan, por lo menos, hasta los ocho años de edad, entonces se entenderá que el proceso de formación de la lateralidad tampoco culmina a los cuatro años. Es 
Alétheia 2014, 2 (1) 33

alrededor de los 6 años, al iniciar la educación primaria, que el niño recién opta por una mano dominante para la realización de la escritura brindando con esto cierta seguridad sobre su lateralidad diestra o zurda, la cual, a los siete u ocho años, ya estará bien definida y el niño optará por una mano, un brazo, un ojo, un oído, una pierna y un pie prioritarios para la realización de sus acciones.

\section{Especialización hemisférica cerebral}

La tasa de individuos zurdos en la población general es del 8 al 10\% aproximadamente, una minoría que le proporcionó al género humano una exitosa variante funcional, ya que se encuentran sobre representados en las ciencias y las artes (PestanaTirado et al., 2004). Esto puede ser explicado a través de las especializaciones que han desarrollado cada hemisferio del cerebro.

Al hemisferio cerebral derecho se le atribuye las habilidades espaciales y visuales (por ejemplo, permite concebir el mundo a través de imágenes, representaciones e intuiciones), la creatividad, la capacidad de síntesis, el talento artístico (especialmente la música), los aspectos básicos del lenguaje verbal y las emociones, por lo que se dice que es el lado emocional y creativo. A través de este hemisferio se reconoce las relaciones entre partes diferentes y las interrelaciones entre cosas y sucesos. Mientras que al hemisferio cerebral izquierdo, se le atribuye habilidades que permite concebir el mundo de una manera lógicoracional, gracias a él se puede ordenar las experiencias, analizarlas y categorizarlas. En este lado del cerebro se encuentran los centros del lenguaje y la escritura, por lo que se le conoce como el centro principal del lenguaje, de la palabra y de los símbolos.

Mercer (1991), refiere que existe suficiente evidencia de que los dos hemisferios difieren en sus papeles en cuanto al aprendizaje, siendo el hemisferio izquierdo el más comprometido en el proceso de palabras y símbolos, utilizándose sobre todo para el desarrollo del pensamiento analítico, secuencial y otros procesos relacionados con el conocimiento; mientras que el hemisferio derecho se ocupa principalmente, de los estímulos no verbales, tales como la imaginería visual y la orientación temporal y espacial. Condemarín (1989, p. 84), también hace referencia a la especialización hemisférica, pero brinda mayores datos sobre su relación con el proceso de aprendizaje:

Los datos actuales no otorgan la exclusiva lateralización de las funciones del lenguaje al hemisferio izquierdo, ya que las observaciones de pacientes con división del cuerpo calloso muestran que el hemisferio derecho conserva funciones a un nivel de cinco años de edad en el análisis sintáctico y su vocabulario auditivo es equivalente al rendimiento de 12 años de edad, aproximadamente. El hemisferio izquierdo está especializado en los aspectos lógicos, sintácticos y ordenados (secuenciales) del lenguaje. La correspondencia grafema-fonema es analizada por el hemisferio izquierdo y las palabras son identificadas usando significados denotativos. El hemisferio derecho incluye las palabras de alta frecuencia de uso, las palabras que son connotativas y que son productoras de imagen. La organización léxica del hemisferio derecho no depende de conceptos verbales o funcionales, son usadas asociaciones visuales estructurales y creativas.

Esto influye significativamente en las acciones que las personas realizan para adquirir conocimientos del medio, por ejemplo, se observa un fenómeno interesante: La persona con predominio del hemisferio izquierdo toma partes separadas, las une, las pone en una relación lógica, de manera que está trabajando desde una parte hacia un todo. La persona con predominio del hemisferio derecho, al contrario piensa en totales, primero observa el total y luego las partes de las cuales está compuesto y así es capaz de descubrir coherencias y soluciones no convencionales.

Aquellos niños con predominio del hemisferio izquierdo piensan y trabajan con símbolos, mientras los niños con predominio del hemisferio derecho se llevan mejor con objetos concretos. Estos últimos aprenden mediante experiencias prácticas, mediante la manipulación y el movimiento de las cosas por lo que necesitan de ejemplos visuales para poder aprender. Resulta así que ambas formas de dominio cerebral corresponden también a un dominio funcional y corporal. Por otrolado, según Geschwind, las funciones 


\section{UNIFÉ - EPG}

del lenguaje están mayoritariamente controladas -u originadas- en el hemisferio izquierdo (en un 90\% de los diestros y entre el 50 y $70 \%$ de los zurdos) sin tener en cuenta el dominio hemisférico (Mercer, 1991).

Es evidente que por esta especialización funcional hemisférica, los zurdos son considerados como personas más sensibles, imaginativas y creativas en comparación a los diestros, aunque también más torpes y menos locuaces que ellos; en relación a esto, diversos investigadores refieren que aunque los individuos zurdos tienen al hemisferio cerebral derecho como hemisferio dominante, y por lo tanto la mano preferente será la izquierda, desarrollando con ella un mayor control motor y por ende la escritura, sin embargo, para las funciones de lenguaje hablado y las otras funciones intelectuales tienen como dominante el hemisferio cerebral izquierdo, al igual que las personas diestras.

Para Pestana-Tirado et al. (2004), en el caso específico de los zurdos, por estar en un mundo derecho (al revés para ellos), desde la niñez se ven sometidos a las presiones adaptativas y como respuesta afortunada desarrollan de manera casi natural y sin esfuerzo, además del dominio de su mano dominante (izquierda), un control desmedido de su mano auxiliar (derecha), con lo que se puede decir que un zurdo domina más rápidamente su mano auxiliar derecha que un diestro su mano auxiliar izquierda.

Lo anterior da una ventaja funcional notable ya que el zurdo podrá desarrollar habilidades que corresponden a los diferentes hemisferios, dándole un rasgo de alta utilidad potencial para el niño, haciéndose menos vulnerable a las lesiones de un hemisferio dominante (Zuliani, 2003).

Por otro lado, existen investigaciones longitudinales en las que se han descrito y correlacionado esta especialización hemisférica con algunos factores del desarrollo del niño, básicos para el aprendizaje escolar.

$\mathrm{Al}$ respecto, Valett (1996) hace referencia a un estudio longitudinal de 390 niños realizado por MacBurney y Dunn en 1976, cuyas edades comprendían entre los tres meses y seis años y medio, en el que se observó que a los seis años y medio el $76 \%$ utilizaba la mano derecha de forma dominante, y sólo el 67\% tenían lateralidad diestra afirmada de mano, pie y ojo, lo que evidencia -para los investigadoresque el $11 \%$ restante tendrían una lateralidad cruzada o contrariada. Además, las puntuaciones obtenidas en las pruebas de lenguaje fueron significativamente mejores en los niños que usaban la mano derecha en las siguientes edades: nueve meses, cuatro años y seis años y medio; y en las pruebas de inteligencia, los diestros consiguieron mejores puntuaciones en las escalas verbal, ejecutiva y total. Por último, los niños cuya lateralización de pie y ojo eran igual a la de la mano (derecho o izquierdo) obtenían puntuaciones significativamente superiores. Con estos datos, Valett sugiere que la consistencia de lateralización refleja integración neuropsicológica y permite un mejor rendimiento académico.

Otros estudios demuestran que existe una relación entre la lateralización cerebral y el aprendizaje de la lectura. Bakker (Bravo, 1999) ha realizado estudios sobre los niños disléxicos, sin embargo, sus resultados también son importantes en el estudio de los niños lectores zurdos. La conclusión a la cual llega es que en el proceso continuo de aprendizaje de la lectura, el niño desarrolla estrategias cognitivas de acuerdo a la etapa -inicial y avanzado- de la lectura en la que se encuentra. Los niños que se encuentran en la etapa inicial procesarían primero la información perceptivovisual (reconoce y discrimina signos gráficos) y después la información verbal (asociada a la pronunciación y al significado), con lo cual se evidencia la acción del hemisferio cerebral derecho, el que decodifica perceptivamente los signos gráficos.

Luego Bakker plantea que en la etapa avanzada, la especialización del hemisferio izquierdo es mayor y por lo tanto el procesamiento del contenido verbal recae básicamente en su acción. En el caso de los niños lectores normales (en su mayoría -como ya se dijo anteriormente- diestros), conforme desarrollan una lectura más avanzada, su dominancia cerebral se manifiesta en las estrategias que van desarrollando, pero en el caso de los zurdos, su hemisferio cerebral dominante sigue siendo el derecho, y aunque los centros del lenguaje se encuentren en el hemisferio izquierdo, 
las estrategias cognitivas utilizadas dependerán de su hemisferio dominante.

En efecto, los primeros estudios sobre niños disléxicos acentuaron la relación de la dislexia con la lateralidad manual zurda, pero en la actualidad se postula que la relación se da con mayor frecuencia en niños con lateralidad manual mixta o inconsistente (Matute, 2001).

\section{Dificultades en la formación de la lateralidad}

A partir de lo expuesto, se puede concluir que el niño debe desarrollar su lateralidad en forma espontánea e iniciar su lateralización con la práctica de ambos lados del cuerpo hasta que defina su lateralidad, pero si un niño mayor a los cuatro años aún no demuestra una preferencia lateral, es recomendable prestar atención a su desarrollo ya que podría tener problemas en su maduración motriz. En otras palabras, el ser humano nace con una organización cerebral establecida y pretender cambiar el predominio de una mano sobre la otra sólo acaba aportando confusión e inseguridad (Zuliani, 2003).

Milicic (2002) también hace referencia a las dificultades que podría tener un niño zurdo en su educación formal, sobretodo en el aprendizaje de la lectura y de los conceptos derecha e izquierda, por lo que sugiere que tanto padres como maestros debieran ser tolerantes con ellos al comienzo de la escolaridad ya que luego superarán rápidamente esta dificultad.

En efecto, investigaciones realizadas en Cuba y recogidas por AUPEC (1996), han revelado que trastornos presentados en la niñez como tartamudez, enuresis nocturna, pesadillas, entre otros, o características como timidez y apatía sin razón aparente, pueden ser ocasionados por la presión familiar y de la sociedad para que estos niños utilicen su mano derecha, cuando en realidad son zurdos. Ante esto, Pestana-Tirado et al. (2004) recuerdan que el dextrismo y la zurdera tienen fundamentos genéticos que la educación y la práctica la acentúan, en cambio el ambidextrismo es aprendido y se convierte en una habilitación manual doble como respuesta adaptativa a las presiones que el medio ejerce sobre el individuo y se considera derivado principalmente de la zurdera.

Por otro lado, la observación directa de niños zurdos permite apreciar que estos niños tienden a fatigarse más rápido al realizar trabajos manuales, sobretodo los que se refieren al uso del papel y lápiz o al corte con tijera; suelen ser más lentos en sus ejecuciones motrices y aparentemente van más despacio, pero en realidad se demoran porque tienen que calcular la dirección de sus trazos, además de cuidar el no ensuciar sus trabajos debido a que su mano (izquierda) sigue los trazos realizados con el lápiz o lapicero, manchando lo escrito, con la consiguiente sensación de suciedad.

\section{Estudios realizados sobre los escolares zurdos}

En cuanto a las investigaciones neurológicas relacionadas al aprendizaje de la lectura, éstas se encuentran muy vinculadas a los estudios realizados con niños disléxicos, los que a su vez, hacen referencia a la zurdera como un factor interviniente, en este sentido, Bravo (1999) hace referencia a los estudios realizados por Naidoo en 1972, donde comparó a niños con retardo en el aprendizaje de la lectura con otros que tenían retardo en la escritura y encontró que ambos grupos tenían como elemento común, un retardo maduracional neurológico y de lenguaje. Este último se hizo evidente solamente cuando los niños habían llegado a la edad de utilizar frases. También encontró déficits en el aprendizaje de las secuencias de los fonemas, pero el hallazgo más significativo para esta exposición, es el que refiere que entre los niños con retardo en la lectura había más porcentaje de niños con zurdera y ambivalencia en el predominio manual, lo que de alguna forma evidencia una mayor tendencia de los niños zurdos a presentar alteraciones para aprender a leer.

En cuanto al aprendizaje de los niños zurdos, especialmente de la lectura, Valett (1996) también hace referencia a algunos trabajos significativos, los que permiten entender la percepción que por muchos años se ha tenido de los zurdos, por ejemplo, cita a Linksz, quien en 1973 resalta la importancia de 


\section{UNIFÉ - EPG}

la decodificación auditiva y del habla en la lectura y escritura, señalando que su desarrollo gradual depende básicamente de una operación única del hemisferio izquierdo. Además hace referencia a que la percepción visual es una habilidad evolutiva en la que la dominancia de uno de los ojos se adquiere mediante su uso, y que en su determinación, un factor esencial; es el uso preferente de una mano, o sea, la dominancia manual, es por esto que Linksz justifica todo esfuerzo razonable dirigido a que los niños zurdos escriban con la mano derecha.

En el Perú, Alvarez (2005)realizó un estudio para conocer la relación entre el autoconcepto y la comprensión lectora en escolares zurdos de segundo y tercer grado de primaria de la ciudad de Lima, y para analizar mejor estos resultados, se les comparó con los resultados obtenidos por escolares diestros de los mismos grados. Los resultados son sumamente interesantes, en la medida que si bien se encuentra que no existe dicha relación entre escolares zurdos, sin embargo, al realizar un análisis de correlación entre los factores que conforman dichas variables, se observa que, a nivel de segundo grado de primaria, la comprensión lectora sí se correlaciona positivamente con el factor apariencia física y atributos (referido a la forma cómo el niño se percibe a sí mismo como persona y a su cuerpo) y con el factor ansiedad (ausencia de indicadores de ansiedad o de problemas de tipo emocional), pertenecientes al autoconcepto; mientras que a nivel de tercer grado, no se encuentra correlación alguna entre la comprensión lectora y los factores del autoconcepto.

Esto puede ser explicado tomando en consideración dos aspectos: a) el primer y segundo grado constituyen el nivel escolar en el cual la lectura y la escritura son los objetivos de enseñanza, lo que no ocurre en el tercer grado, y por ende, se podría decir que tanto el rendimiento escolar general del niño como la presión y carga emocional que lo acompaña, recae sobre estos aprendizajes.En el caso de los alumnos de tercer grado, los objetivos de enseñanza van más allá de la lecto-escritura, se asume que ya saben leer y escribir por lo cual se prioriza la enseñanza de contenidos académicos de los diversos cursos; y b) en el caso específico de los escolares zurdos de segundo grado de primaria, precisamente los factores del autoconcepto que se correlacionan con la comprensión lectora, son aquellos que hacen alusión a sus características físicas y atributos, y a la ansiedad que podría ocasionarle el enfrentarse a las situaciones nuevas o difíciles que les toca vivir.

Con respecto al segundo punto, se podría entender que para el niño zurdo, dicha condición no sólo hace referencia a una preferencia lateral, por el contrario, hace alusión a una condición personal, a la totalidad de su cuerpo, alsí mismo como persona, es él mismo (self) cuando se equivoca al escribir, al leer, al persignarse o al amarrarse los pasadores de los zapatos, etc., y es "cuestionado" por los adultos que se encuentran a su alrededor, ya sean padres, profesores, hermanos, amigos y demás personas. En el fondo, se está "cuestionando" la totalidad de su ser, por lo que surge en él una sensación de inutilidad, de entender que algo malo pasa en él, y por supuesto, eso "malo" sería su condición de zurdo, lo cual no sólo repercute en las tareas donde se hace evidente y palpable su zurdera, sino en todas las acciones que requieran destrezas y habilidades, y entre ellas se encuentra la lectura.

Pero curiosamente, cuando al niño zurdo le va bien en la escuela, el entorno social tiende a resaltar dicha condición de zurdo como el factor que ha permitido el buen desempeño, claro que aquí no estamos diciendo que eso sea cierto, sólo que comúnmente, se tiene la idea que los zurdos son más inteligentes.

En este sentido, tomando en consideración que a) los niños zurdos desarrollan una fortaleza creciente para adaptarse a una sociedad hecha predominantemente para diestros, y b) son niños normales, con capacidades iguales a la de los otros niños, es que se puede entender que ellos logran superar esta situación y son capaces de reestructurar su autoconcepto, encontrándose así, a escolares de tercer grado que diferencian adecuadamente el desarrollo de una habilidad y el concepto que van formando de sí mismos, por lo que se observa precisamente en estos alumnos que no existe ninguna correlación significativa entre la comprensión lectora y los factores del autoconcepto. 
$\mathrm{Al}$ analizar los factores que intervienen en el autoconcepto, se encuentra que entre los escolares zurdos y diestros de segundo grado tampoco existen diferencias significativas, pero sí a nivel de tercer grado, básicamente en los factores relacionados al estatus intelectual y escolar, apariencia física y atributos, y popularidad, a favor de los diestros.

Al respecto, estos resultados podrían ser interpretados tomando en consideración dos condiciones importantes: a) Como menciona Rogers (1994), las primeras experiencias escolares inician el proceso de diferenciación creciente del autoconcepto entre los alumnos, por lo que, aparentemente, durante los primeros años escolares, los niños tienen una imagen más estable sobre sí mismos, los cuales van variando con el transcurso del tiempo, desarrollándose de esta forma, las diferencias individuales; y b) Como refiere Epstein, las presiones adaptativas hacen que el niño zurdo desarrolle mayores estrategias de afrontamiento a fin de superar las limitaciones que encuentra en su vida cotidiana, lo que necesariamente, influirá en la elaboración de su autoconcepto, el cual está compuesto por una serie de conceptos concretos, consistentes y jerárquicamente organizados (Fierro, 1981), que se originan, forman y desarrollan en función a ciertas entidades que le brindan información, como por ejemplo, la familia, la escuela, los amigos, entre otros, además de lo que observa y percibe de sí mismo (Jourard y Landsman, 1988).

En este sentido, los escolares zurdos de segundo grado, pertenecen a este grupo de alumnos que inician el proceso de diferenciación, por lo cual, las diferencias que podría tener en relación a los diestros, aún no se dan, en cambio, a nivel de tercer grado, los escolares zurdos ya han asimilado una serie de informaciones sobre sí mismos y a la vez, son concientes de las limitaciones que tienen para desempeñar algunas tareas, por lo que sus autoconceptos presentan diferencias en determinados factores en comparación con los escolares diestros, aunque esto no represente diferencias en el autoconcepto general.

\section{Conclusiones}

La lateralidad es una condición inherente a la persona humana la cual está condicionada por factores genéticos, que implican la especialización de los hemisferios cerebrales, sin embargo, el proceso que involucra su adecuada adquisición se ve influenciado por factores sociales. La lateralidad permite reconocer cuál es la dominancia cerebral de la persona, la cual se evidencia en la habilidad que demuestra un hemicuerpo sobre el otro, en las tareas de control motor fino, entre otras.

La adecuada lateralización puede influir en el desarrollo socioafectivo del niño, en la medida que para realizar las distintas tareas o actividades de acuerdo a su nivel de desarrollo se requieren de instrumentos o medios que, en su mayoría, han sido elaborados para las personas diestras, por lo que el zurdo debe desarrollar mayores patrones de comportamiento adaptativo para poder salir adelante. Este es el problema que surge en la educación, es decir, aún no se está preparado para atender a estos niños y aceptar la posibilidad de ciertas demoras en la adquisición de las habilidades lectoras y escritoras, lo cual hace que sean considerados como niños flojos o poco hábiles, lo que en muchos casos los puede conducir al fracaso escolar.

\section{Referencias}

AUPEC - Agencia Universitaria de Periodismo Científico (1996). Discriminación contra los Zurdos. Recuperado de http://www.univalle. edu.co/\%7Eaupec/AUPEC/octubre96/ zurdos.html

Alvarez, C. (2005). Comprensión lectora y autoconcepto en escolares zurdos de segundo y tercer grado de primaria. (Tesis de magister inédita). Universidad Ricardo Palma, Lima, Perú.

Bravo, L. (1999). Lenguaje y Dislexias. Enfoque Cognitivo del Retardo Lector. México D.F., México: Alfaomega.

Cebec (2004). ¿Qué significa ser zurdo?. Recuperado de http://www.zurdos.cl/que_significa.html

Condemarín, M. (1989). Lectura Correctiva y Remedial. Santiago de Chile, Chile: Ediciones S. M. Chile. 
38 UNIFÉ - EPG

Fierro, A. (1981). Lecturas de Psicología de la Personalidad. Madrid: Alianza Editorial.

Holder, M. (2004). Primate Handedness and Brain Lateralization Research. Recuperado de http:// www.indiana.edu/ primate/index.html

Jourard, S., y Landsman, T. (1988). La Personalidad Saludable: El punto de vista de la Psicología Humanística. México D. F., México: Editorial Trillas.

Matute, E. (2001). Neuropsicología de la Lectura. En Alcazar, V. y Gumá, E. (Editor). Texto de Neurociencias Cognitivas. México D. F., México: El Manual Moderno.

Mercer, C. (1991). Dificultades de Aprendizaje 1. Barcelona, España: Ediciones CEAC.

Milicic, N. (2002). Guía para padres. El niño zurdo. Revista virtual Miprimer Icarito. 23. Recuperado de http://www.miprimericarito.cl/2002/08/16/ sicologa02.htm.

Pestana-Tirado, R., Moreno, L., y Di Filippo, A. (2004). Dominio Motor y destreza. La verdadera esencia del cirujano. En Revista Colombiana de Cirugía. 19(4). Recuperado de http://www.encolombia. com/medicina/cirugia/Ciru19404-Dominio. htm

Quintans, S. (2005). Huellas de otros tiempos. Suplemento del Diario Virtual El Clarín. Recuperado de http://www.clarin.com/suplementos/ viajes/2005/01/23/v-01011.htm

Rogers, C. (1994). Psicología Social de la Enseñanz̧a. Madrid: España: Visor Distribuciones S. A.

Segovia, C. (2004). Albergue de Náufragos Zurdos. Recuperado de http://www.inclusiondigital. net/albergue/zurdos/index.html

Valett, R. (1996). Dislexia. Barcelona, España: Grupo Editorial Ceac.

Vallés, A. (1995). Técnicas de Velocidad y Comprensión Lectora. Madrid, España: Escuela Española, S.A.

Zuliani, L. (2003). El uso de ambas manos posibilita un desarrollo mayor. Revista Médica Pediátrica. Órgano Oficial de la Sociedad Colombiana de Pediatría. 38(2). Recuperado de http:// www.encolombia.com/medicina/pediatria/ pediatria38303-crianza1.htm 